\title{
INHALT DES VIERUNDACHTZIGSTEN UND FÜNFUNDACHTZIGSTEN BANDES
}

\author{
I. ABTEILUNG
}

H. HUNGER, Anagrammatismos - Paragrammatismos. Das Spiel mit den Buchstaben . . .

D. R. REINSCH. Die editio princeps eines Auszuges aus der Alexias Anna Komnenes aus dem Jahr 1562; ein unabhängiger ṫberlieferungsträger . . . . . . . . . . . . . .

B. L. FONKIČ/F. B. POLJAKOV, Markos Eugenikos als Kopist. Zur Tätigkeit eines Gelehrtenkreises an den Konstantinopolitaner Skriptorien im ersten Drittel des 15. Jahrhunderts

G. CHR. HANSEN, Germanos, der findige Leser . . . . . . . . . . . . . . . . . . .

C. GERBINO, Appunti per una edizione dell'agiografia di Lentini . . . . . . . . . .

H. KAHANE + /R. KAHANE, Justinian's Credo in Western Medieval Literature . . . . . .

H. E. J. CowdREY, Pope Victor and the Empress A. . . . . . . . . . . . . . . . .

K. P. MATSCHKE, Zum Anteil der Byzantiner an der Bergbauentwicklung und an den Bergbaucrträgen Südosteuropas im 14. und 15. Jahrhundert . . . . . . . . . . . . .

F. MADDEN, The Fires of the Fourth Crusade in Constantinople. 1203-1204; A Damage As-

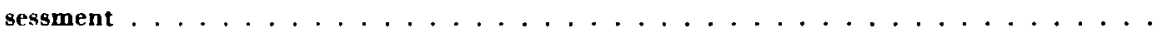

N. THIERRY, Importance du culte de Saint Jean Prodrome en Cappadoce. A propos des absides

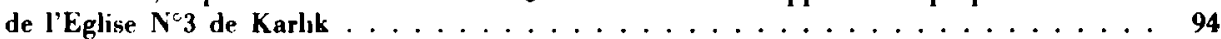

W. BERSCHIN, Griechisches in der Klosterschule des alten St. Gallen . . . . . . . . . . 329

W. KASSEL, Aristophanisehes in einer byzantinischen Invektive des 15. Jahrhunderts . . 341

E. FOLLIERI, Ancora una nota sul Christus Patiens . . . . . . . . . . . . . . . . . 343

E. VELKOVSKA, Another manuscript of Joseph Melendytes? . . . . . . . . . . . . 347

B. MONDRAIN, Copistes et collectionneurs de manuscrites grecs au milieu du XVIe siècle: . 354

A. P. KAZHDAN/A.-M. TALBOT, Women and iconoclasm . . . . . . . . . . . . . . . . . . 392 J. KODER, „Zeitenwenden“. Zur Periodisierungsfrage aus byzantinischer Sicht . . . . . . . 409

G. GUILLOU/C. ROGNONI, Une nouvelle fondation monastique dans le Theme de Calabre (1053-1054)

G. CAVALlo, Mezzogiono svevo e cultura greca

S. V. BLIZNJUK, Die Venezianer auf Zypern im 13. und in der ersten Hälfte des 14. Jahrhunderts ..............................

D. JACOBY, Silk in western byzantium before the fourtb crusade . . . . . . . . . . . .

O. KRESTEN, Oktateuchprobleme: Bemerkungen zu einer Neuerscheinung . . . . . . . . sist

\section{ABTEILUNG}

CHR. WILDBERG, John Philoponus' Criticism ... besprochen von P. W. SHARPLES . . . 117 The Life of Michael the Synkellos ... ed. by M. B. CUNNINGHAM ... besprochen von A. BER-

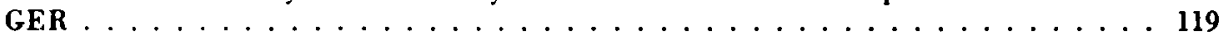

Gnomica Basileensia ed. by J. F. KINDSTRAND ... besprochen von H. G. NESSELRATH 120

P. NITSCHE, Nicht an die Griechen glaube ich ... besprochen von F. B. POLIAKOV . . . 121

U. MOENNIG, Die spätbyzantinische Rezension und Zur Uberlieferungsgeschichte des mittelund neugriechischen Alexanderromans ... besprochen von W. J. AERTS . . . . . . . . . 122

Iohannis Cantacuzeni: Refutationes duae ... ed. E. VOORDECKERS/F. TINNEFELD $\ldots$ besprochen von G. FATOUROS . . . . . . . . . . . . . . . . . 125

O. BERGGÖTZ, Der Bericht des Marsilio Zorzi ... besprochen von V. EPP . . . . . . . . . 126

A. CHA UVOT, Procope de Gaza ... besprochen von C. CAPIZZI . . . . . . . . . . . . . . 128

M. MAAS, John Lydus and the Roman Past ... besprochen von L.-M. GUNTHER . . . . . 129

C. IMBER, Ottoman Empire ... besprochen von C. ZACHARIADOU . . . . . . . . . . . . 130

CHR. GIZEWSK I, Zur Normativität und Struktur der Verfassungsverhältnisse ... besprochen von F. GORIA . . ............................ 
P. M. STRÄSSLE, Der internationale Schwarzmeerhandel ... besprochen von L. MAKSIMOVIĆ 135

D. NICOL, A biographical Dictionary ... besprochen von G. MAKRIS . . . . . . . . . . 137

A. BERGER, Untersuchungen zu den Patria Konstantinupoleos ... besprochen von A. KUL.

ZER . . . . . . . . . . . . . . . . . . . . . . . 138

A. NEGEV, The architecture of Mampsis I + II ... besprochen von P. GROSSMANN . . . 139

F. W. DEICHMANN, Ravenna ... Bd. II, 3. Teil ... besprochen von J. M. SPIESER . . . . 143

J. GUYON, Le cimetière aux deux lauriers ... besprochen von E. JASTRZEBOWSKA . . 146

W. HAHN/W. E. METCALF, Studies in Early Byzantine Gold Coinage ... besprochen von

M.Salamon . . . . . . . . . . . . . . . . . . . 148

The Oxford Dictionary of Byzantium, hrsg. von A. P. KAZHDAN u. a. ... besprochen von

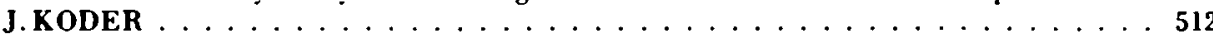

R. A. KASTER, Guardians of Language ... besprochen von C. THEODORIDIS . . . . . . 515

The letters of loannes Mauropous ... ed. by A. KARPOZILOS ... besprochen von E. TRAPP 518

I. ROCHOW, Byzanz im 8. Jahrhundert in der Sicht des Theophanes ... besprochen von

A. BERGER . . . . . . . . . . . . . . . . . . . . . . 519

PTOCHOPRODROMOS, Einfuihrung, kritische Ausgabe, deutsche Ubersetzung, Glossar besorgt von Hans Eideneier ... besprochen von W. J. AERTS . . . . . . . . . . . . 519

KRASOPATERAS, Kritische Ausgabe der Versionen des 16.-18. Jahrhunderts, besorgt von Hans Eideneier ... besprochen von M. HINTERBERGER . . . . . . . . . .

C. POCHERT, Die Reimbildung in der Spät- und Postbyzantinischen Volksliteratur ... bespro-

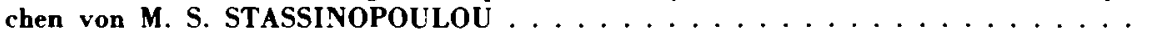

Katalog der griechischen Handschriften der Osterreichiscben Nationalbibliothek Teil 3/3: hrsg. von H. HUNGER/W. LACKNER ... besprochen von J. IRIGOIN . . . . . . . . . . 525

K. P. TODT, Kaiser Johannes VI. Kantakuzenos und Islam ... besprochen von F. TINNEFELD 526

F. VAN DE PAVERD, St. John Chrysostom, The homilies on the statues ... besprochen von G. WEISS . . . . . . . . . . . . . . . . . . . . . . . . . . . 528

Diversorum Postchalcedonensium Auctorum Collectanea I. Pamphili Theologi Opus, hrsg. von

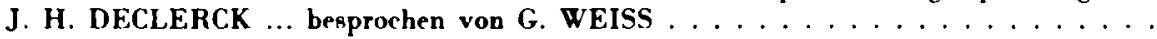

Maximi Confessoris Quaestiones ad Thalassium II. Edd. C. LAGA/C. STEEL ... besprochen von G. WEISS . . . . . . . . . . . . . . . . . . 530

K. P. CHRISTOU, Byzanz und die Langobarden ... besprochen von J. JARNUT . . . . . 531

H.-J. KUHN, Die byzantinische Armee im 10. und 11. Jahrhundert ... besprochen von P. M. STRÄSSLE . . . . . . . . . . . . . . . . . . . . . . 532

Prosopographisches Lexikon der Palaiologenzeit. Erstellt von E. TRAPP ... besprochen von D. M. NICOL . . . . . . . . . . . . . . . . . . . . 533

H. BELTING, Bild und Kult. Eine Geschichte des Bildes ... besprochen von J. GAUS . . . 533

K. ENGLERT, Der Bautypus der Umgangskirche ... besprochen von E. C. CONSTANTINIDES 539

R. WISSKIRCHEN, Das Mosaikprogramm von S. Prassede in Rom ... besprochen von

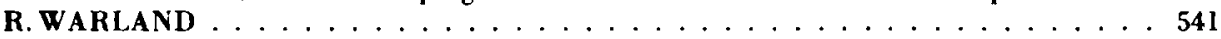

C. JOLIVET-LEVY, Les églises byzantines de Cappadoce $\ldots$ besprochen von J. ALBANI . 543

o. DEMUS, Die byzantinischen Mosaikikonen I. ... besprochen von V. H. ELBERN . . . . 545

J. LOWDEN, Illuminated Prophet Books. A Study of Byzantine Manuscripts ... besprochen

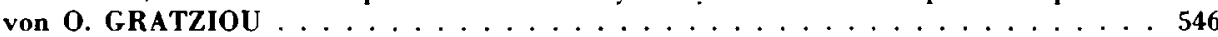

J. NESBITT/N. OIKONOMIDES, Catalogue of Byzantine Seals at Dumbarton Oaks and in

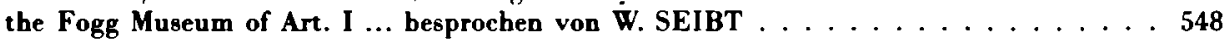

\section{ABTEILUNG}

Bibliographische Notizen, Mitteilungen und Nachrufe $\ldots \ldots \ldots \ldots \ldots \ldots$. . . . . $\ldots 2,551$ 